\title{
ANÁLISE FÍSICO - QUÍMICA DO LEITE CRU PROVENIENTE DE UMA PROPRIEDADE RURAL DO PONTAL DO PARANAPANEMA
}

Jaqueline Nascimento, Nayara Pereira Rocha

Universidade do Oeste Paulista, Curso de Química Bacharelado, Presidente Prudente, SP. E-mail: jaqueline@unoeste.br, nayara pereira14@hotmail.com.

\section{RESUMO}

Do ponto de vista do controle de qualidade, o leite e os derivados lácteos estão entre os alimentos mais testados e avaliados, principalmente devido a importância que representam na alimentação humana e á sua natureza perecível. Os testes empregados para avaliar a qualidade do leite fluído constituem normas em todos os países. O produtor além de consumir esse leite em natura também o usa para fazer derivados sem prévia análise ou informação do mesmo. O objetivo do estudo foi verificar a qualidade físico-química do leite cru proveniente da propriedade, comparando com a legislação vigente, informando ao produtor sobre sua produção leiteira e qualidade de suas matrizes. Para tanto foram feitas análises de $\mathrm{pH}$, determinação de gordura, determinação de estabilidade ao alizarol, determinação da densidade e determinação de sacarose. Os resultados obtidos foram satisfatórios, todas as amostras estavam dentro dos limites aceitos pela legislação vigente, estando dentro do padrão de qualidade.

Palavras-chave: Leite Cru. Gordura. Propriedade rural. Parâmetros de leite. Legislação.

\section{PHYSICAL - CHEMICAL ANALYSIS OF CRUDE MILK FROM A RURAL PROPERTY OF PONTAL DO PARANAPANEMA}

\begin{abstract}
Of the point of view of quality control, milk and your dairy derivatives are among the most tested and evaluated foods, mainly due to the importance they represent in human feeding and its perishable nature. The tests used to evaluate the quality of fluid milk constitute norms in all countries. The producer besides consuming this milk in natura also uses it to make derivatives without previous analysis or information of the same. The objective of the study was to verify the physical-chemical quality of raw milk coming from the property, comparing with the current legislation, informing the producer about its milk production and quality of its matrices. Therefore, were realized analysis of $\mathrm{pH}$, fat determination, determination to alizarol stability, determination of density and determination of sucrose were carried out. The obtained results were satisfactory, all samples were within the limits accepted by the current legislation, being within the quality standard.
\end{abstract}

Keywords:Raw Milk. Fat. Rural property. Parameters of milk. Legislation. 


\section{INTRODUÇÃO}

O leite está entre os seis produtos mais importantes da agropecuária brasileira, ficando atrás somente de produtos como café beneficiado e arroz. O agronegócio do leite e seus derivados desempenham um papel relevante no suprimento de alimentos e na geração de emprego e renda para a população (EMBRAPA, 2005).

Segundo o IBGE em 2014, a produção de leite no país foi de 35,17 bilhões de litros, representando um aumento de $2,7 \%$ em relação à registrada no ano anterior. O Brasil ocupou, em 2014, a segunda posição mundial em relação ao efetivo de vacas ordenhadas, de acordo com o Departamento de Agricultura dos Estados Unidos (United States Department of Agriculture USDA), ficando atrás apenas da Índia, que possui o maior rebanho de bovinos do mundo (MILK POINT, 2015).

O preço médio nacional do litro do leite foi de $\mathrm{R} \$ \mathbf{0 , 9 6}$, gerando um valor de produção de $\mathrm{R} \$ 33,78$ bilhões em 2014. O maior preço médio foi encontrado na Região Nordeste, $\mathrm{R} \$ 1,11$, enquanto o menor, na Região Norte, $\mathrm{R} \$ 0,82 \mathrm{O}$ valor do produto incentiva à produção de leite por pequenos produtores (MILK POINT, 2015).

No Pontal do Paranapanema cerca de $80 \%$ dos produtores comercializa o leite com os laticínios instalados nos municípios, bem como em outros laticínios da região e dos estados vizinhos (MAZZINI, 2007). O assentamento Che Guevara, localizado no Pontal do Paranapanema, de acordo com o ITESP, tem mais de $25 \%$ de sua área destinada a pecuária leiteira. O peso que as atividades vinculadas à pecuária leiteira têm na ocupação da área ocorre no valor gerado para os produtores do local (DIVERSITAS, 2000).

Apesar da produção de leite estar crescendo na região, os produtores não se preocupam muito com a qualidade do leite em questão, pois para eles o que importa é o custo/beneficio que esse ramo da agropecuária lhes proporciona. Os dados indicam que $100 \%$ da renda familiar provêm das atividades realizadas no lote. (MAZZINI, 2007). As vacas da região normalmente, não são de raça, ou de inseminação, são matrizes leiteiras simples, originadas do cruzamento de várias raças. Nessa área há um simples acompanhamento de um técnico do ITESP, ou INCRA, ao qual vem à propriedade rural orientar sobre vacinas, registros e notas desses animais, sem nenhum outro acompanhamento de como cuidar do rebanho, da produção de leite ou das qualidades do mesmo. O leite cru proveniente da propriedade além de ser vendido é consumido pelos integrantes da residência, e utilizado para fazer derivados sem nenhuma análise prévia do mesmo, apenas com o cozimento do leite; (DIVERSITAS, 2000).

Devido à grande importância do leite na nutrição e do modo em que é consumido o objetivo do estudo foi analisar a qualidade físico química do leite cru proveniente de uma propriedade rural do Pontal do Paranapanema.

\section{METODOLOGIA}

Foram coletadas amostras de 5 matrizes da propriedade rural em dias alternados conforme proposto em frascos âmbares tratados termicamente por processo de autoclave.

As matrizes foram selecionadas de acordo com suas idades, quantidade de crias e média do porte do bezerro.

A última vacina aplicada foi na data de 22 de maio de 2017 contra febre aftosa.

A alimentação é no pasto com a qualidade de grama brachiária humidícula, água e com suplementação mineral de sal mineral (Matsuda fós leite) e sal branco.

O leite é retirado uma vez ao dia, pela manhã. O bezerro fica com a matriz somente meio período e logo após já é apartado para que se consiga a formação de leite nas glândulas mamárias. 


\begin{tabular}{c|c|c|c}
\hline \multirow{2}{*}{ Matriz } & \multicolumn{2}{|c|}{ Detalhes das matrizes } \\
\\
$1^{\circ}$ & Porte Matriz & Crias & Porte Bezerro \\
$2^{\circ}$ & Nova & $2^{\circ}$ & Médio \\
$3^{\circ}$ & $2^{\circ}$ & Novo \\
$4^{\circ}$ & Nova & $5^{\circ}$ & Velho \\
$5^{\circ}$ & Nova & $1^{\circ}$ & Velho \\
& Velha & $6^{\circ}$ & Velho \\
\hline
\end{tabular}

Fonte: Dados experimentais

Todas as análises foram realizadas na Universidade do Oeste Paulista, Bloco Q, Laboratório de Alimentos.

pH do Leite

Análise realizada em temperatura ambiente com pHmetro de bancada devidamente calibrado. Sendo colocada a amostra em béqueres diferentes, numerados e com volume de $50 \mathrm{~mL}$ cada.

\section{Densidade}

A densidade foi analisada em temperatura ambiente, sendo a amostra de leite colocada em provetas diferentes, numeradas e com volume de $250 \mathrm{~mL}$ cada. E inserido na proveta vagarosamente o termolactodensímetro da marca Incoterm $^{\circledR}$ 5784.1. Feita a leitura da temperatura e da densidade e corrigido para a leitura de $15^{\circ} \mathrm{C}$ conforme tabela de correção de densidade disponível no Laboratório.

Gordura por método de Gerber

Os resultados foram lidos em butiromêtros devidamente limpos, separados e numerados, com o seguinte procedimento: Adição no butiromêtro de $10 \mathrm{~mL}$ de ácido sulfúrico PA leite $\mathrm{D}=1825$, mais $11 \mathrm{~mL}$ da amostra de Leite e $2 \mathrm{~mL}$ de álcool amílico, fechado com rolha até a marcação agitados vagarosamente para a mistura total do leite no ácido e no álcool e então colocados para centrifugar com velocidade média de $5000 \mathrm{rpm}$ por um tempo de 5 minutos contados em cronometro de bancada. Finalizada a centrifugação o resultado foi lido na graduação do butiromêtro e conforme os números e necessidades ajustou-se a rolha para cima ou para baixo.

Acidez por método de Alizarol

Colocado em 5 béqueres devidamente limpos e numerados de 1 a $5,10 \mathrm{~mL}$ de amostra e 10 $\mathrm{mL}$ de alizarol e analizado a cor de cada reação e comparando com a tabela de Fernando Rodrigues, 2005 
Tabela 2. Padrão Cor Reação Alizarol

\begin{tabular}{cc}
\hline Cor & Interpretação \\
\hline Vermelho=lilás (tijolo) & $\begin{array}{c}\text { Sem coagulação - Leite } \\
\end{array}$ \\
Vermelho Castanho & $\begin{array}{c}\text { Coagulação fina - Leite normal partindo } \\
\text { para o ácido- acidez de } 19 \text { a } 21^{\circ} \mathrm{D}\end{array}$ \\
\hline Amarelo coagulado & Coagulação mais espessa- Leite ácido- \\
& Acidez superior a 21 ${ }^{\circ} \mathrm{D}$ \\
\hline Violeta sem coagulação & Leite alcalino (ou fraudado)- Acidez \\
& abaixo de $16^{\circ} \mathrm{D}$ \\
\hline
\end{tabular}

Fonte: FERNANDO RODRIGUES, 2005.

Identificação de sacarose com resorcina

Foram medidas as amostras em provetas de $15 \mathrm{~mL}$ separadas, transferidas para béqueres de $50 \mathrm{~mL}$, adicionado a cada um deles $1 \mathrm{~mL}$ de ácido clorídrico e $0,1 \mathrm{~g}$ de resorcina, agitado e colocado em banho Maria por 5 minutos. Sendo assim analisada a coloração de cada uma das amostras. Pois em meio ácido a resorcina condensa-se com as aldoses formando um composto na coloração rosa, ou seja, é um indicador de sacarose. (ADOLFO LUTZ, 2008).

\section{RESULTADOS}

Depois de realizado todos os cálculos, os resultados foram dispostos na forma de tabela para melhor visualização do mesmo. Nesta tabela será apresentado o resultado das análises de Alizarol. Para determinação dos resultados foram utilizadas quantidades iguais de amostra e alizarol (1:1). A tabela abaixo apresenta os valores encontrados após as análises.

Tabela 3. Determinação de Alizarol

\begin{tabular}{|c|c|c|c|c|c|}
\hline Data & Matriz 1 & Matriz 2 & Matriz 3 & Matriz 4 & Matriz 5 \\
\hline $26 / 06 / 2017$ & $\begin{array}{l}\text { Vermelho/ } \\
\text { Lilás }\end{array}$ & $\begin{array}{l}\text { Violeta sem } \\
\text { coagulação }\end{array}$ & $\begin{array}{l}\text { Vermelho/lilá } \\
\text { s }\end{array}$ & Vermelho/lilás & $\begin{array}{l}\text { Vermelho/Cast } \\
\text { anho }\end{array}$ \\
\hline $28 / 06 / 2017$ & $\begin{array}{l}\text { Vermelho/ } \\
\text { Lilás }\end{array}$ & $\begin{array}{l}\text { Violeta sem } \\
\text { Coagulação }\end{array}$ & $\begin{array}{l}\text { Amarelo com } \\
\text { coagulação }\end{array}$ & $\begin{array}{l}\text { Vermelho } \\
\text { Castanho/coagul } \\
\text { ação fina }\end{array}$ & Vermelho/lilás \\
\hline $30 / 06 / 2017$ & $\begin{array}{l}\text { Vermelho/ } \\
\text { castanho }\end{array}$ & $\begin{array}{l}\text { Amarelo com } \\
\text { coagulação }\end{array}$ & $\begin{array}{l}\text { Amarelo com } \\
\text { coagulação }\end{array}$ & Vermelho/lilás & Vermelho/lilás \\
\hline
\end{tabular}

Para a verificação de densidade Foi realizado análise com termolactodensimetro e corrigido com tabela de correção padrão de densidade. A tabela 4 logo abaixo apresenta os valores encontrados.

Tabela 4. Determinação de densidade $(\mathrm{g} / \mathrm{mL})$

\begin{tabular}{cccccc}
\hline Data & Matriz 1 & Matriz 2 & Matriz 3 & Matriz 4 & Matriz 5 \\
\hline $26 / 06 / 2017$ & 1,0279 & 1,0279 & 1,0279 & 1,0279 & 1,0290 \\
\hline $28 / 06 / 2017$ & 1,0263 & 1,0263 & 1,0322 & 1,0290 & 1,0290 \\
\hline $30 / 06 / 2017$ & 1,0271 & 1,0282 & 1,0282 & 1,0293 & 1,0293 \\
\hline
\end{tabular}

Fonte: Próprio autor 
Para a quantificação de gordura foi usado o método de Gerber e dispostos os resultados na tabela abaixo.

Tabela 5. Determinação da gordura por método de Gerber

\begin{tabular}{cccccc}
\hline Data & Matriz 1 & Matriz 2 & Matriz 3 & Matriz 4 & Matriz 5 \\
\hline $26 / 06 / 2017$ & $4,1 \%$ & $4,2 \%$ & $4,5 \%$ & $5,7 \%$ & $3,7 \%$ \\
\hline $28 / 06 / 2017$ & $4,1 \%$ & $3,5 \%$ & $1,5 \%$ & $4,7 \%$ & $3,7 \%$ \\
\hline $30 / 06 / 2017$ & $4,1 \%$ & $3,1 \%$ & $4,7 \%$ & $4,0 \%$ & $4,0 \%$ \\
\hline \multicolumn{7}{c}{ Fonte: Próprio autor } & &
\end{tabular}

Para a análise de $\mathrm{pH}$ foi utilizado pHmetro de bancada devidamente calibrado. A tabela 6 logo abaixo apresenta os valores de $\mathrm{pH}$ para cada uma das amostras

Tabela 6 - Determinação $\mathrm{pH}$

\begin{tabular}{cccccc}
\hline Data & Matriz 1 & Matriz 2 & Matriz 3 & Matriz 4 & Matriz 5 \\
\hline $26 / 06 / 2017$ & 6,80 & 6,98 & 6,85 & 6,84 & 6,75 \\
\hline $28 / 06 / 2017$ & 6,82 & 6,89 & 6,75 & 6,77 & 6,70 \\
\hline $30 / 06 / 2017$ & 6,86 & 6,93 & 6,78 & 6,77 & 6,85 \\
\hline
\end{tabular}

Fonte: Próprio autor

Para averiguação de adultérios foi empregado a análise com resorcina, onde indica a reação com sacarose ou não. Os resultados foram expressos na tabela a seguir.

Tabela 6. Análise de Resorcina

\begin{tabular}{|c|c|c|c|c|c|}
\hline Data & Matriz 1 & Matriz 2 & Matriz 3 & Matriz 4 & Matriz 5 \\
\hline $26 / 06 / 2017$ & Não reagente & Não reagente & Não reagente & Não reagente & Não reagente \\
\hline $28 / 06 / 2017$ & Não reagente & Não reagente & Não reagente & Não reagente & Não reagente \\
\hline $30 / 06 / 2017$ & Não reagente & Não reagente & Não reagente & Não reagente & Não reagente \\
\hline
\end{tabular}

\section{DISCUSSÃO}

De acordo com a EMBRAPA (2005), a densidade do leite é em média 1,032 $\mathrm{g} / \mathrm{mL}$ podendo ter uma variação entre $1,023 \mathrm{~g} / \mathrm{mL}$ e $1,040 \mathrm{~g} / \mathrm{mL}$. Considerando um leite de $3 \%$ de gordura a média da densidade seria em torno de $1,025 \mathrm{~g} / \mathrm{mL}$, enquanto um leite de $4,5 \%$ de gordura deveria ter em média de $1,0277 \mathrm{~g} / \mathrm{mL}$. Tendo em vista esses parâmetros, todas as matrizes estão dentro da densidade considerada normal e na visão do controle de qualidade significa que a matriz tem um percentual aceitável de cada componente do leite.

Comparando com o que diz EMBRAPA (2005), que o pH do leite fresco apresenta uma reação ligeiramente ácida, variando entre 6,6 e $6,8 \mathrm{com}$ uma média de 6,7 a $20^{\circ} \mathrm{C}$ ou a $6,6 \mathrm{em}$ $25^{\circ} \mathrm{C}$. Somente leite com glândulas mamarias com mastite (doença que causa inflamação) o pH pode ficar alcalino podendo ficar entre 7,3 e 7,5. No Caso de quatro matrizes considerando a temperatura ambiente de $24^{\circ} \mathrm{C}$ o pH ficou dentro dos limites de variação, somente a matriz número 2 que foi 0,1 a mais que o limite, um valor ainda considerável de variação.

$\mathrm{Na}$ análise para identificação de sacarose com resorcina Mendes (2010), diz que na adição de sacarose (ou açúcar domestico), o produtor pretende mascarar uma adulteração com água para que a densidade relativa volte ao normal, e o teste de resorcina pretende identificar esse tipo de mascaração. Ao entrar em contato com ácido e aquecimento o indicador resorcina identifica a presença de sacarose e fica na coloração vermelha. Foi feito uma amostra teste, onde foi adicionado açúcar domestico no leite e seguido o procedimento de análise, onde o leite ficou rosa 
escuro para comparação com as amostras normais. Em comparação com essa descrição nenhuma das amostras foi identificado com sacarose adicionada.

Na descrição de Rodrigues (2005), a análise de alizarol é uma determinação rápida e aproximada da acidez do leite por calorimetria podendo ser feita na própria propriedade rural ou na bancada de recepção do laticínio onde a interpretação de resultados do teste de alizarol deve ser levado em conta a formação ou não de coagulação e a coloração formada comparada com parâmetros pré estabelecidos.

Comparando matriz por matriz, vemos que as de bezerro mais velho tem a acidez maior, visando a desmama da cria e o termino do ciclo do leite.

De acordo com EMBRAPA (2005) a variação de gordura do leite está entre 3,5\% e 5,35\% isso levando em consideração a diferença de raças, alimentação das matrizes, estágio de lactação e estação do ano. Verificado cada uma das análises de gordura as matrizes estão todas dentro do padrão de qualidade, exceto pela $3^{\circ}$ matriz no segundo dia de análise, que pode ser explicado pela idade da matriz e idade do bezerro que está na fase de desmama. De uma forma geral podemos apontar ao produtor que o percentual de gordura está satisfatoriamente bom para a época do ano (Inverno).

\section{CONCLUSÃO}

A partir das tabelas foi possível avaliar que as amostras estão dentro dos limites aceitáveis. Dessa forma considera-se que as matrizes da propriedade rural estão dentro do padrão de qualidade.

\section{REFERÊNCIAS}

DIVERSITAS. Assentamento Che Guevara, 2000. Disponível em:

<http://diversitas.fflch.usp.br/files/06-Assentamento\%20Che\%20Guevara.pdf>. Acesso em: 15 set. 2017.

EMBRAPA. Agronegócio do leite, 2005. Disponível em: <http://www.agencia.cnptia.embrapa.br/Agencia8/AG01/arvore/AG01_193_21720039246.html>. Acesso em: 15 set. 2017.

INSTITUTO ADOLFO LUTZ. Normas Analíticas do Instituto Adolfo Lutz. v. 1: Métodos químicos e físicos para análise de alimentos, 3. ed. São Paulo: IMESP, 1985. p.217. Disponível em: <http://www.ial.sp.gov.br/resources/editorinplace/ial/2016_3_19/analisedealimentosial_2008.pd f> Acesso em 15 set. 2017

MAZZINI, ELIANE DE JESUS TEIXEIRA. Assentamentos rurais no Pontal do Paranapanema - SP: uma política de desenvolvimento regional ou de compensação social?. 2007. 311 f. Dissertação (mestrado) - Universidade Estadual Paulista, Faculdade de Ciências e Tecnologia, 2007. Disponível em: <https://repositorio.unesp.br/handle/11449/96775>. Acesso em: 15 set. 2017.

MENDES, CAROLINA DE GOUVEIA ET AL. Ánalises físico-químicas e de fraude do leite informal comercializado no município de Mossoró - RN. Ciência Animal Brasileira, [S.I.], v. 11, n. 2, p. 349356, jun. 2010. ISSN 1809-6891. Disponível em: <https://www.revistas.ufg.br/vet/article/view/1146/8161>. Acesso em: 15 set. 2017.

MILK POINT. IBGE: Produção de leite cresceu 2,7\% em 2014; Sul tornou-se a maior região produtora. Disponível em: 
<http://www.milkpoint.com.br/cadeia-do-leite/giro-lacteo/ibge-producao-de-leite-cresceu-27em-2014-sul-tornouse-a-maior-regiao-produtora-97326n.aspx>. Acesso em: 15 set. 2017

RODRIGUES, F. Manual de bancada para laboratório (BPL) fábrica de queijos. Disponível em: $<$ http://docplayer.com.br/9517540-Manual-de-bancada-para-laboratorio-bpl-fabrica-de-queijosfernando-rodrigues-parte-ii.html>>. Acesso em: 15 set. 2017. 